\title{
Un guide SHF pour la petite hydroélectricité
}

\author{
A small hydro SHF handbook
}

A l'initiative de la Société hydrotechnique de France, une commission spécialisée comprenant à la fois des maitres d'œuvre, des fabricants de machines et des installateurs vient de réaliser un Guide pour la conception, la réalisation, la mise en service et l'exploitation des petits aménagements hydroélectriques. (270 pages).

Cette commission, présidée par M. P. Morand (Compagnie nationale du Rhône), était animée par MM. G. Feillée (Electricité de France) et C. Le Plomb (Compagnie nationale du Rhòne).

Destiné aux personnes ou organisations peu familiarisées avec la petite hydroélectricité, le guide, dont le sommaire figure en annexe, comprend deux parties bien distinctes.

La première partie, qui constitue le guide proprement dit, couvre la totalité de l'itinéraire à suivre pour réaliser une installation depuis l'esquisse du projet jusqu'à sa réalisation, sa mise en service et son exploitation. Une série de démarches successives, conduiront le lecteur en fonction de divers critères de choix, soit à une réalisation effective, soit à l'abandon du projet.

Le travail tient compte de la relation essentielle entre les domaines d'application et l'impact économique et social de l'énergie produite dans le contexte local.

Au préalable, afin de bien situer les conditions du placement de l'énergie, sont exposées diverses notions pratiques relatives à l'utilisation de l'énergie ainsi que les contraintes liẻes à sa qualité.

Puis, sont abordées la méthodologie générale conduisant à une première estimation de la faisabilité d'un projet et la façon de conduire les études (études préliminaires, étude de faisabilité et avant projet détaillé).

S'agissant des matériels, un développement particulier détaille les éléments à prendre en considération pour les garanties à demander aux fabricants ainsi que pour les contrôles permettant de s'assurer de la bonne qualité des matériels fournis, étant entendu que les vérifications de ces garanties et les contrôles imposés ne doivent pas entraîner un coût prohibitif.

Des indications sont ensuite données pour aider à la rédaction de l'appel d'offres (avec un exemple de découpage en lots des équipements électromécaniques) et pour faciliter la comparaison des offres reçues.

Enfin, sont soulignés conseils et mises en garde en vue d'éviter des perturbations dans le déroulement du chantier ainsi que dans la prise en charge de l'installation après le départ des constructeurs.

Un lexique sommaire précise la définition de la terminologie technique utilisée dans le guide lors- qu'elle n'est pas précisée directement dans le texte.

La deuxième partie du document consiste en une série de dix annexes qui développent certaines données évoquées à l'intérieur des chapitres de la première partie. En fonction de ses propres connaissances des problèmes liés à la minihydroélectricité, l'utilisateur du guide aura donc à s'y référer, ou non.

Ces annexes ont :

- soit un caractère purement technique lié à la détermination du projet depuis l'inventaire des ressources hydroélectriques, les besoins d'énergie, le dimensionnement des ouvrages et le choix des machines:

- soit un caractère économique et administratif (étude financière et étude d'impact) permettant d'arriver à la réalisation de l'appel d'offres;

- la dernière annexe donne une liste d'ouvrages récents relatifs à la minihydroélectricité.

\section{Plan du guide}

Préambule

Ch. I - Présentation du guide - Processus

Ch. II - Présentation des ouvrages

Ch. III - Orientation méthodologique générale pour l'étude d'un projet

Ch. IV - Placement de l'énergie

Ch. V - Conduite des études

Ch. VI - Nature et vérification des garanties

Ch. VII - Appel d'offres

Ch. VIII - Comparaison des offres

Ch. IX - Exécution et réception des ouvrages

Ch. $\mathrm{X}-$ Exploitation des ouvrages

Terminologie

Annexes

1 - Inventaire des ressources hydroélectriques

2 - Prévisions du développement de la consommation d'ènergie électrique

3 - Études sur le terrain

4 - Dimensionnement général des ouvrages

5 - Choix des groupes

6 - Études économique et financière

7 - Étude d'impact

8 - Appels d'offres

9 - Feuilles documentaires pour les petites installations hydrauliques

10 - Bibliographie

En vente à la Société hydrotechnique de France (199. rue de Grenelle, 75007 Paris. Tél. 47.05.13.37) au prix de $250 \mathrm{~F}$. 
Le Président remercie d’abord M. FEILLÉE de sa présentation des travaux menés par la Commission sur la Minihydroélectricité et des indications qu'il a données sur le rapport fait par $M$. TRANAIN qui n'a pu participer à la séance.

II remercie ensuite M. Le PLOMB de son exposé sur les principes et le contenu de louvrage élaboré.

Le Président. - Avant d'ouvrir la discussion, si nous voulions résumer certains aspects essentiels, nous pourrions dire que la volonté du groupe de travail, sous l'impulsion de ses animateurs. a été avant tout d'essayer d'éviter tout d'abord au maitre d'ouvrage, quel qu'il soit, de se trouver engagé dans un processus dont il ne sache plus sortir si, à un moment donné. il apparaissait que l'ouvrage projeté aurait, pour des raisons soit techniques, soit économiques, étẻ difficilement réalisable, voire mẻme irréalisable. Par conséquent, il était important de marquer des étapes, voire même des possibilités de retour en arrière d'un certain processus qui les traduise, de manière à pouvoir s'arrêter ou "corriger le tir " si. à un certain moment. le résultat d'études relativement légères et, dans la progression de la préparation du projet, d'études de plus en plus lourdes montrait que l'on est engagé sur une mauvaise voie.

D'autre part, nous avons cherché aussi à éviter. et ceci au bénéfice réciproque du maître d'ouvrage et des constructeurs, que des maitres d'ouvrage mal informés n'en demandent trop. ce qui a évidemment pour premier effet d'accroitre les coúts. mais qui peut aussi introduire des distorsions de concurrence. ceci afin qu'on n'aille pas demander parfois des choses farfelues. peut-être mème simplement en matière d'essais, á des constructeurs. Jai le souvenir davoir dvu 9 énormes pompes, selon la volonté du maitre d'ouvrage et de son ingénieur. dans un pays africain, passer en rang d'oignon sur le banc d'essai du CETIM de Nantes, qui était le seul à pouvoir les accueillir en France. Toutes ces pompes sont allées de Chartres à Nantes de Nantes à Chartres, puis de Chartres à Rouen pour le simple plaisir de les avoir essayées une par une, alors qu'elles étaient rigoureusement identiques et relevaient de modèles parfaitement classiques du constructeur. C'est aussi ce genre de chose que nous avons voulu. sinon éviter. du moins atténuer en faisant en sorte qu'un maître d'ouvrage aussi bien informé que possible n’aille pas demander des choses que nous pourrions qualifier de superfétatoires.

Nous allons maintenant aborder la discussion. Qui demande la parole?

M. Giravlt. - Est-ce que ce Guide va donner lieu à l'établissement d'un cahier des charges simplifié ?

M. Le Président. - Je me tourne vers les responsables du groupe de travail, mais aussi vers le Président BaNAL.

L'idée initiale était de faire un document; on avait parlé de normalisation au début. puis nous nous sommes aperçu en fait que vouloir normaliser les petites centrales hydroélectriques était peut-être beaucoup trop ambitieux et en même temps que ce n'était peut-être pas faisable.

De la mẻme façon, en ce qui concerne un cahier des charges simplifié, je m'interroge un peu. parce que, dans un cahier des charges, il y a un certain nombre de principes, qui ressortent d'ailleurs assez clairement de la deuxième partie du Guide et surtout de ses annexes: mais de là à vouloir présenter un modèle. alors que nous nous adressons à des maîtres d'ouvrage extrèmement hétérogènes. puisque nous nous adressons à des maîtres d'ouvrage de tous les pays du monde, qui ont des traditions techniques très variées: les traditions techniques françaises, anglo-saxonnes, allemandes, etc. en matière de spécifications ne sont pas les mêmes.

Nous avons cherché à définir des principes qui aident à ce que les choses soient comparables. De là à déboucher sur un cahier des charges simplifié, je ne sais pas ce qu'en pense le Président BANAL, mais je craindrais que ce ne soit peut-être un peu à cóté de notre objectif.
M. BANAL - Je répondrai volontiers que si vraiment un besoin se manifeste. la Société Hydrotechnique de France n'éludera pas ses responsabilités ni son désir d'être utile.

Je pense qu'un des intérêts de l'information qui est lancéc aujourd'hui par ce Guide est justement de provoquer les rèactions des lecteurs. Les auteurs ont dit qu'il contenait des erreurs, des coquilles, etc. Que l'on nous dise les corrections a faire, nous en seront très contents. Mais je pense que l'on peut aller plus loin. et dire que s'il y a des compléments à apporter. de nouvelles directions dans lesquelles on puisse aller, pourquoi pas? La SHF écoutera à partir d’aujourd hui les réactions. les commentaires qui vont être donnés sur ce Guide.

Puisque jai la parole. je dirai que j'ai une petite critique à faire: dans le Guide. il n'est pas question des mini-usines marémotrices: c'est un sujet qui n'a pas été abordé et qu'on aurait pu traiter. Je me demande s'il ne serait pas intéressant de faire un complément à l'usage des personnes des régions littorales qui voudraient faire des mini-usines maremotrices.

M. Le Président. - Je crois quil y a lả un champ daction fort intéressant, mais qui risque de soulever parfois localement des polémiques.

M. GaUtheron. - Une petite usine marémotrice avait été envisagée à St Pierre et Miquelon, mais la marée étant trés faible, de l'ordre du mètre. l'opération n'ètait pas économiquement envisageable.

M. Monition. - Le projet en tant quopération de petite marémotrice, opération encouragée par la Commission des communautés Européennes / D G 17. n’est pas abandonné. II pourrait être transféré en Bretagne où les marées sont de plus grande amplitude. Par contre, des aẻrogénérateurs pourraient étre proposés pour St Pierre et Miquelon doté de ressources éoliennes importantes.

M. FEILLEEE - Le guide ne traite pas des petits groupes expérimentaux (vagues, courants marins, etc.).

M. Le Président. - De toute façon, ce Guide se voulant essentiellement un instrument d'utilisation pratique, il traite d'aspects qui sont du domaine en cours. Les projets qui en sont encore au stade de la recherche, voire du développement technologique n'avaient pas leur place, compte tenu de l'esprit dans lequel ce document a été préparé.

M. GIRAULT. - Electrobras est en train de développer un guide du méme type au Brésil. N'y a-t-il pas une coopération possible à envisager ?

M. Le Président. - Bien que ne connaissant pas le projet, la S.H.F. accepterait d'y participer et demande à chacun de participer au maximum à la diffusion internationale du guide.

M. ARGENSON. - Le guide ne fait-il pas double emploi avec ceux publiés aux U.S.A. par des Universités?

\section{Le Présidemt. - La bibliographie a été faite.}

M. FEILLEEE. - Tous les guides n'ont pas le même but. Par exemple, celui de I'UNIDO est plutôt orienté vers la recherche de financement. Les guides américains sont destinés à des centrales plus importantes jusqu'à 20 ou $30 \mathrm{MW}$ et donnent plus des coûts que des méthodes à suivre. De même. le projet de la CEI se limite aux matériels. Ces divers ouvrages sont des compléments et ne font donc pas double emploi avec notre guide.

M. Le Président, - Je crois que, de toute façon, ce qu'il est important de noter, c'est que nous avons voulu aborder le problème en considérant l'opération d'aménagement dans son ensemble; c'est peut-être ce qui fait le plus l'originalité du guide qui vous est présenté aujourd'hui. 